\title{
New Options of Apheresis in Renal Diseases: How and When?
}

\author{
Jolanta Korsak Zofia Wańkowicz \\ Military Institute of Medicine, Warsaw, Poland
}

\section{Key Words}

Plasmapheresis - Cryofiltration - LDL apheresis - Therapeutic plasma exchange $\cdot$ Immunoadsorption $\cdot$ American Society of Apheresis

\begin{abstract}
The 100-year anniversary of the first experimental apheresis performed by John Abel on uremic dogs in 1914 provides the opportunity for discussion on the current state of classic apheresis as well as technological progress and clinical experiences with its new options presented in the world literature in the last 15 years, such as the following: double filtration, plasma adsorption and immunoadsorption, leuko- and cytapheresis and low-density lipoprotein apheresis. In our review, we highlight the potential limitations for further development of those highly promising techniques, such as the following: the lack of multicenter, controlled clinical studies; insufficient knowledge of the mechanisms of those techniques and last but not least, the restricted access to apheresis, caused both by high expenditure and organizational negligence, even in highly developed countries. Special attention was paid to the most recent recommendations by the American Society of Apheresis in primary and secondary renal diseases, which are the subject of our professional interest.

(c) 2015 S. Karger AG, Basel
\end{abstract}

\section{Introduction}

Therapeutic apheresis (TA) is an extracorporeal blood purification method employed in treating those of the renal diseases, pathogenesis and clinical course of which depend on the accumulation of the immune complexes, allo- or autoantibodies, cryo- or immunoglobulins in the patient's plasma. Removal of those substances by way of apheresis promises either a total remission (apheresis as a primary treatment) or improved results of an immunosuppressive treatment (apheresis as a supporting treatment). What is more, apheresis causes an immunological modulation effect that is both suppressive and stimulating in its nature. Suppressive nature of the treatment manifests itself through reduced quantity of pathological substances (antibodies, immune complexes and immunoglobulins). The stimulating properties, on the other hand, may be observed in reactivation of the cellular immunological response. Different techniques of apheresis are implemented depending on the size of a particle which needs to be removed: therapeutic plasmapheresis (TP), known also as plasma exchange (PLEX), therapeutic plasma exchange (TPE) or TA; low-density lipoprotein (LDL) apheresis (when removing LDL lipoproteins); double filtration; plasma- and immunoadsorption (IA), cryofiltration or cytapheresis [1].

\section{KARGER}

E-Mail karger@karger.com

www.karger.com/bpu
(C) 2015 S. Karger AG, Base

0253-5068/15/0413-0001\$39.50/0
Zofia Wańkowicz

Military Institute of Medicine

ul. Szaserów 128

PL-04-141 Warszawa, Mazowieckie (Poland)

E-Mail zwankowicz@ wim.mil.pl 
Table 1. Therapeutic apheresis techniques and methods in renal diseases [6]

\begin{tabular}{|c|c|c|}
\hline Technique & Method & Type of pathogen removed \\
\hline Plasma exchange & $\begin{array}{l}\text { Centrifugation or filtration method of plasma } \\
\text { separation - requires supplemental fluids }\end{array}$ & $\begin{array}{l}\text { Antibodies, immunological complexes, other } \\
\text { pathological proteins }\end{array}$ \\
\hline Double filtration & $\begin{array}{l}\text { Centrifugation or filtration method of plasma } \\
\text { separation complemented with refiltration, } \\
\text { requires supplemental fluids }\end{array}$ & $\begin{array}{l}\text { Immunological complexes, autoantibodies, other } \\
\text { pathological proteins }\end{array}$ \\
\hline Cryofiltration & $\begin{array}{l}\text { Centrifugation or filtration method of plasma } \\
\text { separation complemented with re-filtration and } \\
\text { cooling, does not require supplemental fluids }\end{array}$ & Cryoproteins \\
\hline Plasma adsorption & $\begin{array}{l}\text { Centrifugation or filtration method of plasma } \\
\text { separation, adsorption on phenylalanine, } \\
\text { tryptophan or polymyxin B-filled columns }\end{array}$ & $\begin{array}{l}\text { Anti-DNA antibodies, myeloperoxidase, ANCA, IgG } \\
\text { immunoglobulins, lupus-like anticoagulant, endotoxins, } \\
\text { cytokines, C-reactive protein, immunological complexes, } \\
\text { TNFa, VEGF, macrophage inflammatory protein }\end{array}$ \\
\hline Immunoadsorption & $\begin{array}{l}\text { Protein A, anti-IgG Fc antibodies adsorption } \\
\text { (i.e. dextran sulfate) }\end{array}$ & Antibodies, protein complexes \\
\hline LDL apheresis & $\begin{array}{l}\text { Chemical compounds adsorption (tryptophan, } \\
\text { polyacrylate) }\end{array}$ & LDL lipoprotein \\
\hline Cytapheresis & Centrifugation method of plasma separation & CD8 lymphocytes, CD4, activated platelets, granulocytes \\
\hline
\end{tabular}

First attempts at therapeutic application of plasmapheresis were conducted in 1952 on patients with hyperviscosity syndrome in the course of multiple myeloma [2]. Application of this treatment in renal diseases was postulated after the report from Lockwood et al. [3] on its effectiveness in rapidly progressive glomerulonephritisRPGN (with the presence of anti-glomerular basement membrane antibodies). Plasmapheresis was combined with immunosuppressive treatment before oliguria occurred and while the creatinine concentration in plasma remained below $6.5 \mathrm{mg} / \mathrm{dl}$.

In 2001, Andre Kaplan noted the possibility of adopting TA in primary renal diseases like RPGN associated with the presence of anti-neutrophil cytoplasmic antibodies (ANCA) and IgA nephropathy, as well as secondary renal diseases like paraproteinemias, especially multiple myeloma, cryoglobulinemia, systemic lupus erythematosus (SLE), systemic vasculitis and thrombotic microangiopathy. Plasmapheresis was also advocated as a procedure reducing the side effects of the immunosuppressive treatment [4].

In our center, we began performing TA in 1989, and in 1998, we published our first clinical experiences on applying apheresis in the treatment of RPGN [5]. Up to now, we have performed 2016 interventions; of those, 169 were on patients suffering from various renal diseases.
This paper reviews world experience of the last 15 years regarding new technological options of classic apheresis and their clinical utility in the treatment of autoimmune renal diseases.

\section{New Options of Apheresis in Renal Diseases: How?}

Decision on the use of apheresis in renal diseases must be followed by a secondary choice of a suitable technique, as it determines the effectiveness of the intervention as well as the risk of side effects. Techniques of TA as employed in various renal diseases are summarized in table 1 [6].

PLEX is the most commonly used apheresis technique in the treatment of renal diseases. Selective techniques of pathogen elimination were first implemented by the end of the 20th century, some of which are the following: immunoadsorption plasmapheresis (IAPP); double filtration plasmapheresis (DFPP); low-density lipoprotein apheresis (LDL) and cryofiltration [6,7].

The nature of each listed plasmapheresis technique lies in the separation of blood components and in the removal of pathological factor. Some of the techniques fulfil these requirements using chemical substances such as adsorbents as well as patient's circulating blood volume supplementation. 
Classic TPE separates and removes the pathogen by way of centrifugation or filtration methods. Centrifugation method utilizes the differences in specific gravities and sedimentation rates of particles in relation to other blood components. Filtration method, on the other hand, utilizes the variety in sizes of particles passing through microporous membrane. Centrifugation method has a more varied application, that is, it allows not only the separation of plasma, but also separates the cellular components of blood. The filtration method is used mostly to separate plasma and less often to remove its pathological components [8].

Regardless of the technique, effectiveness of the treatment depends on some basic conditions. First and foremost, the substance undergoing removal must occur in the blood and have a molecular weight above $15,000 \mathrm{Da}$ or an appreciable affinity for plasma proteins. Substances with large volume of distribution are not subject to elimination by way of apheresis due to their much lower concentration in the plasma compared to the other fluid areas of the organism. Secondly, the knowledge of the transcapillary escape rate is necessary. This rate differs depending on the molecular weight of plasma components. For example, immunoglobulin M (IgM) exists mostly in the intravascular space, immunoglobulin $\mathrm{G}$ (IgG) is distributed almost evenly between both spaces, and albumins are prevalent in the intravascular space $[9,10]$.

Finally, one of the most relevant conditions of the apheresis efficacy is knowledge of the pathways through which pathological substances are eliminated. Each of the substances occurring in the plasma undergoes a natural degradation process with a distinctive velocity. Recognizing the rate of this degradation is important in the clinical practice. It influences the decision-making process both in inclusion of apheresis into the treatment, as well as the frequency and duration of the intervention. Theoretically, it is postulated that during the TP intervention the exchange of substances between intra- and extravascular spaces is inconsiderable; thus, we are dealing with unicompartmental structure. In this arrangement, any substance present in the plasma has a determined initial concentration, which decreases during the apheresis procedure with time and accordingly to the volume of PLEX $[9$, 10].

Each type of apheresis treatment is conducted in the extracorporeal circulation with the use of cell separators. Patients' blood is taken venously and subjected to centrifugation or filtration, which results in the separation of the blood into red cells and component rich plasma, out of which the specific particles are then extracted (LDL li- poproteins, immunological complexes and antibodies). Remaining morphotic blood components and plasma or blood cells suspended in the plasma substituting fluid are transfused back to patient. Extracorporeal blood flow takes place in sterile, disposable separation kit that is compatible with the employed separator. Blood collected through the separator is bound by an anticoagulant solution, most commonly disodium citrate, in a ratio of $1: 13$ blood compounds. Citrate blocks the blood clotting cascade through inhibition of calcium-related platelets and clotting factors activity. Oftentimes, heparin is used as an additional precaution. Its advantage is the possibility of reducing the citrate dosage during the intervention, hence decrease in risk of low calcium concentration in blood serum-related complications [11].

Separators with intermittent or continuous flow are most commonly used in clinical practice. Intermittent flow separators isolate the plasma in cycles of blood collection, plasma separation and re-transfusion of remaining blood elements. This technical solution is based on the fact that the volume of separated plasma and the duration of intervention on patient's hematocrit values are interdependent. Lower the hematocrit, higher the volume of blood taken during one cycle. Apheresis with intermittent flow lasts relatively long (4-6 h), which causes substantial fluctuations in extracorporeal circulation blood volume as well as patients' blood volume $[10,12]$. In separators with continuous flow, the plasma is constantly isolated from the blood cells, which results in lower volume of patients' blood in extracorporeal circulation and lack of significant disturbances in the circulating blood volume. New generation separators make apheresis more precise - observed loss of RBCs and platelets is significantly lower $[13,14]$.

During the apheresis carried out with filtration method, blood taken from the patient is combined with anticoagulant solution and passed through the filtration membrane with pore diameter $0.2-0.65 \mu \mathrm{m}$. This allows the separation of plasma from most of the pathological substances it usually contains [10]. Majority of the new filters use the hollow-fiber technology wherein blood flows inside the filter through a complicated tubing system made of permeable pore membrane, which forms a sieve that separates the plasma [15]. Using the filtration method of plasmapheresis entails considerably longer duration of each intervention and lower biocompatibility of the membranes, which means in comparison to centrifugation method, increased risk of allergic reactions. The additional inconvenience lies in the high frequency with which the heparin is used during treatment. Overexposure to 
this particular anticoagulant may cause the heparin-induced thrombocytopenia (HIT) syndrome [15].

Aside the aforementioned methods, there also exist selective methods of eliminating the undesirable substances from the plasma. Apheresis treatment performed through such a method is divided into 3 stages: (1) plasma is separated from cellular components of blood using centrifugation or filtration method, (2) the isolated plasma once again undergoes a separation through additional filters of hollow-fiber technology and (3) 'cleansed' plasma and cellular blood components are then returned to the patient's bloodstream. For the treatment of renal diseases, the following selective methods are also used: cascade filtration and double filtration - remove particles with high molecular weight; immunoadsorption - allows the separation and removal of antibodies and protein complexes; plasma adsorption - removes lipoproteins and large particles such as fibrinogen, C-reactive protein; cryofiltration - isolates cryoglobulins; cytapheresis - isolates lymphocytes, granulocytes or active platelets.

Cascade filtration, a type of selective plasma purification technique is also known as differential membrane filtration. It is a technique based on the disparities in molecular weight of the removed particles. During the first stage of this treatment, plasma is separated from the morphotic blood components using standard apheresis filter or centrifugation method. Next, plasma undergoes second filtration through a membrane with pores of smaller diameter. This allows the removal of particles bigger than albumin, such as immunoglobulins, lipoproteins and immunological complexes. Cascade filtration is also employed as a substitute to TPE for removal of antibodies in autoimmunological renal diseases. However, cascade filtration as a technique less selective than TPE carries certain risks. It causes simultaneous removal of particles larger than the antibodies, such as fibrinogen, $\alpha-2$ macroglobulin or IgM immunoglobulins, which may influence the rheological properties of blood [11].

Cryofiltration is a modified technique of cascade filtration. The separated plasma is cooled down to below $25^{\circ} \mathrm{C}$ in order to provoke the formation of pathological aggregates of molecules present in the plasma. This way the size of particles increases, making the second stage of the treatment - re-filtration - considerably easier. This technique is employed in selective removal of cryoglobulins and immunological complexes used in the treatment of cryoglobulinemia. Two types of cryofiltration are used: (1) removal of cryoglobulins precipitating in the temperature of $4^{\circ} \mathrm{C}$ and (2) removal of cryogel (anticoagulant complex consisting of fibrinogen, fibronectin, fibrin deg- radation products, cold insoluble proteins and heparin core) conducted in the temperature of $2-10^{\circ} \mathrm{C}[15,16]$.

IA based upon the chromatography affinity principle is highly selective and specific. This technique consists of chemical reactions between chemically reactive ligands bound to the surface of the adsorbent and pathological plasma components. Available immunoadsorbents contain staphylococcal A protein or immobilized sheep antigens used for extraction of human IgG immunoglobulin and immunological complexes [11]. IA is a more selective technique than PLEX. It lowers the concentration of IgG antibodies in bloodstream down even to $95 \%$ of the initial value as soon as after 2 interventions. During the classic PLEX, the concentration of those antibodies never falls below $68 \%$ of the initial value $[8,11]$. Protein $\mathrm{A}$, a component of Staphylococcus aureus cellular membrane, showing a high affinity to IgG, strongly binds the immunoglobulins IgG1, IgG2, IgG4 slightly less IgG3, while IA of immunoglobulins IgGM and IgGA does not exceed $30 \%$ of the output value. The adverse effect of IA might be leukocytostatis, which leads to generalized vasculitis with or without glomerulonephritis $[17,18]$. In IA as adsorbents chemical compounds, like tryptophan or polyacrylate, which lower the concentration of LDL lipoprotein, may be also used. Dextran sulfate is employed in IA combined with hemodialysis or hemofiltration in patients suffering from severe sepsis complicated by acute kidney injury (AKI). Plasma separated with filtration technique undergoes further separation on the adsorptive column filled with dextran sulfate in order to remove interleukins (IL-1, IL-5, IL-6, IL-7, IL-8, IL-10, IL-12, IL-16, IL-18), TNF- $\alpha$ and C-reactive protein. 'Cleansed' plasma and its cellular components are then subject to hemodialysis. Furthermore, in treatment of AKI complicated septic shock refractory to standard treatment may be utilized continuous veno-venous hemodialysis with high cut-off membrane factor $(\geq 60 \mathrm{kDa})$ for average and highmolecular pathological substances (i.e. cytokines responsible for the shock). High cut-off membranes, thanks to their ability to remove myoglobin, can also be used in the treatment of post-traumatic rhabdomyolysis complicated with AKI [19-21].

TA is well tolerated even by patients in bad general condition. The side effects of this treatment were observed in circa $4.75 \%$ of all interventions, wherein the frequency was higher in the case of apheresis where frozen plasma from different donors was used as a substitution fluid [22]. The frequency of those events differs depending of the apheresis technique employed in the treatment and is a direct result of a contact between the patient's 
blood and the apheresis equipment, a change of plasma composition and a reduced concentration of drugs used in the treatment. Most common among them is a symptomatic hypocalcemia - a result of choosing citrate as an anticoagulant. As this compound binds calcium ions, it causes an oversensitivity of neuromuscular system. This reaction may occur in patients with hypoproteinemia or in those who are undergoing apheresis using calcium-deficient substitute fluids. Citrate reacts firstly with ionized calcium and only afterwards moves onto the proteinbound calcium. Other undesirable effects of apheresis are the following: a possible fluid imbalance (hypo- or hypervolemia), coagulopathy and dilution thrombocytopenia (dilution or mechanical destruction), typical for the PLEX and being a result of errors in substitute fluids usage. In case of intensive treatment, the reduction in immunoglobulin concentration can be easily observed. This, especially in correlation with immunosuppressive therapy may lead to immunodeficiency syndrome [23, 24].

PLEX may cause significant fluctuations in pharmacokinetics and the concentration of albumin binding drugs. Apheresis may result in decrease of the concentration of antibiotics and anticonvulsants. Therefore, starting patients on apheresis requires precise calculation of drug pharmacokinesis and administration in relation to the schedule of apheresis procedures.

Risk of death on apheresis is estimated, depending on patients population, from $1 / 1,000$ to $3 / 10 \mathrm{k}$. It should be noted though, that this evaluation reflects the course of underlying disease rather than the risk associated with the treatment alone. The most common causes of death related to apheresis are the disorders of neuromuscular and pulmonary systems, anaphylactic reactions and blood vessels perforation during the implantation of a catheter into the central vein or hemorrhages [22].

\section{New Options of Apheresis in Renal Diseases - When?}

Last 15 years have brought about a rise in new options of TA, such as IA, double filtration, LDL apheresis, cryofiltration and cytapheresis, used in the treatment of both primary and secondary autoimmune renal diseases. Those methods, more selective and safer than the classic TA, are potentially more efficient in removal of pathogens, thus reducing the intoxication of the patient's organism. What is more, contrary to classic TA, they do not require the restoration of large volumes of physiological substances such as albumins or supplementation with fluids, like fresh frozen plasma. In fact, in plasma adsorp-

New Options of Apheresis in Renal Diseases tion method in which the loss of basic plasma proteins is inconsiderable the substitution of fluids and albumins is unnecessary, which also makes this method less risky than classic TA when it comes to allergic reactions [25].

Since 1986, the American Society of Apheresis (ASFA) has published systematic reviews of TA applications (termed the Special Issue for Apheresis Guidelines). Since 2007 , this special Issue is updated every 3 years [26]. The data is organized into 4 categories (I-IV) and graded for strengths of recommendations and quality of published evidence according to Guyatt et al. [27]. In 2013, the most recent sixth edition of the guidelines was published under the supervision of Schwartz et al. [28]. It consists of 78 illnesses and clinical conditions, which meet the ASFA criteria for currently employed TA procedures, such as the following: TPE, erythrocytapheresis, RBC exchange, thrombocytapheresis, leukocytapheresis, extracorporeal photopheresis, IA, LPL apheresis, adsorptive cytapheresis and rheopheresis.

Similar recommendations appropriated for Japanese patients, published by Nakanishi et al. [25] in 2014 take to account 27 illnesses and clinical states resistant to conventional therapy, in which TA or its options, including the frequency and duration of the procedure were prescribed by the Japanese National Health Fund. In Poland, each reference center for this procedure assigns the appropriate indications for each TA option in accordance to the ASFA classification and own experience.

In January 2014, the article by Shaz et al. [29] entitled 'How we developed and use the American Society for Apheresis guidelines for TA procedures' introduced the practical aspects of applying the ASFA recommendations into clinical practice.

In table 2, we have gathered those of the ASFA 2013 recommendations which pertain to application of TA and its options in renal diseases.

With reference to data presented in table 2 , it seems prudent to remind how the ASFA differentiates categories and recommendations. The ASFA puts clinical indications for performance of the appropriate TA procedure in categories I to IV with recommendations $1-\mathrm{A}, \mathrm{B}, \mathrm{C}$ and 2-A, B, C.

Category I consists of diseases in which apheresis is a standard and widely accepted method of treatment.

Category II consists of diseases in which apheresis is accepted as a supplementary or secondary method of treatment.

Category III consists of diseases in which apheresis is employed as a treatment method, but its effectiveness is not sufficiently documented or the published results are 
Table 2. 2013 ASFA category and recommendation grades

\begin{tabular}{|c|c|c|c|}
\hline Disease & $\begin{array}{l}\text { Apheresis } \\
\text { treatment }\end{array}$ & $\begin{array}{l}\text { ASFA } \\
\text { category }\end{array}$ & $\begin{array}{l}\text { Recommendation } \\
\text { grade }\end{array}$ \\
\hline \multicolumn{4}{|l|}{ ANCA associated RPGN (polyangiitis, Wegener's granulomatosis) } \\
\hline Dialysis dependent & TPE & I & $1 \mathrm{~A}$ \\
\hline Diffuse alveolar hemorrhage & TPE & I & $1 \mathrm{C}$ \\
\hline Dialysis independent & TPE & III & $2 \mathrm{C}$ \\
\hline \multicolumn{4}{|l|}{ Anti-glomerular basement membrane disease (Goodpasture's syndrome) } \\
\hline Dialysis dependent & TPE & III & $2 \mathrm{~B}$ \\
\hline Diffuse alveolar hemorrhage & TPE & I & $1 \mathrm{C}$ \\
\hline Dialysis independent & TPE & $\mathrm{I}$ & $1 \mathrm{~B}$ \\
\hline Catastrophic anti-phospholipid syndrome & TPE & II & $2 \mathrm{C}$ \\
\hline Cryoglobulinemia, severe/symptomatic & TPE & $\mathrm{I}$ & $2 \mathrm{~A}$ \\
\hline Focal segmental glomerulosclerosis, recurrent after transplantation & TPE & I & $2 \mathrm{~A}$ \\
\hline \multicolumn{4}{|l|}{ Hemolytic uremic syndrome, atypical } \\
\hline Complement gene mutation & TPE & II & $2 \mathrm{C}$ \\
\hline Factor $\mathrm{H}$ autoantibodies & TPE & I & $2 \mathrm{C}$ \\
\hline MCP mutations & TPE & IV & $1 \mathrm{C}$ \\
\hline \multicolumn{4}{|l|}{ Hemolytic uremic syndrome, infection associated } \\
\hline Shiga toxin associated & TPE & IV & $1 \mathrm{C}$ \\
\hline Streptococcus pneumoniae associated & TPE & III & $2 \mathrm{C}$ \\
\hline \multicolumn{4}{|l|}{ Henoch-Schönlein purpura, severe } \\
\hline Crescentic glomerulonephritis & TPE & III & $2 \mathrm{C}$ \\
\hline Severe extrarenal disease & TPE & III & $2 \mathrm{C}$ \\
\hline \multicolumn{4}{|l|}{ IgA nephropathy } \\
\hline Crescentic glomerulonephritis & TPE & III & 2B \\
\hline Chronic progressive & TPE & III & $2 \mathrm{C}$ \\
\hline Immunocomplex RPGN & TPE & III & $2 \mathrm{C}$ \\
\hline Myeloma cast nephropathy & TPE & II & $2 \mathrm{~B}$ \\
\hline Nephrogenic systemic fibrosis & TPE & III & $2 \mathrm{C}$ \\
\hline \multicolumn{4}{|l|}{ Paraproteinemic demyelinating polyneuropathies } \\
\hline \multirow[t]{2}{*}{ IgG/IgA } & TPE & $\mathrm{I}$ & $1 \mathrm{~B}$ \\
\hline & IA & III & $2 \mathrm{C}$ \\
\hline \multirow[t]{2}{*}{$\operatorname{IgM}$} & TPE & I & $1 \mathrm{C}$ \\
\hline & IA & III & $2 \mathrm{C}$ \\
\hline Multiple myeloma & TPE & III & $2 \mathrm{C}$ \\
\hline Post transfusion purpura & TPE & III & $2 \mathrm{C}$ \\
\hline \multicolumn{4}{|l|}{ Renal transplantation } \\
\hline Desensitization due to donor-specific HLA antibodies, living donor & TPE & $\mathrm{I}$ & $1 \mathrm{~B}$ \\
\hline Desensitization due to donor-specific HLA antibodies, deceased donor & TPE & III & $2 \mathrm{C}$ \\
\hline Antibody-mediated rejection & TPE & $\mathrm{I}$ & $1 \mathrm{~B}$ \\
\hline Sepsis with multiorgan failure & TPE & III & $2 \mathrm{~B}$ \\
\hline \multicolumn{4}{|l|}{ Systemic lupus erythematosus } \\
\hline Nephritis & TPE & IV & $1 \mathrm{~B}$ \\
\hline Severe extrarenal manifestations & TPE & II & $2 \mathrm{C}$ \\
\hline Thrombotic thrombocytopenic purpura & TPE & I & $1 \mathrm{~A}$ \\
\hline
\end{tabular}


inconclusive. In those instances, apheresis may be applied only as a last chance treatment.

Category IV consists of diseases in which apheresis was documented to be completely ineffective, but the treatment may be conducted as a part of clinical trial and requires explicit consent from the patient.

With respect to categories presented above, the following recommendations and their grading was introduced. Recommendation 1 means a strong recommendation, 2 means a weak recommendation, wherein each of them contains 3 quality of evidence grades.

A - high quality evidence - is based upon the results of sufficiently large prospective, randomized clinical studies.

B - moderate quality evidence - is based upon the results of randomized clinical trials of lower quality or high quality observational studies.

C - low quality evidence - is based upon properly conducted observational studies or - very low quality evidence - series of cases or expert opinions.

In our center, we interpreted the individual ASFA categories in relation to renal diseases as follows.

\section{ASFA Category I}

TA should be a first-line treatment method applied as an exclusive therapy or jointly with immunosuppressive treatment. It should be noted that in the category I, grades of recommendation are highly varied, which means that although TA is strongly recommended, the quality of evidence and scientific publications differ. Therefore, it is possible that the intended effect may not occur. The acquisition of further evidence may therefore influence the recommendations and change them considerably.

Example of such evolution is ANCA-associated RPGN, in which TP is categorized accordingly: dialysis dependence as category I/1 A; diffuse alveolar hemorrhaging, as category I/1C; dialysis independence, as category III/2C. On the other hand, RPGN in the course of Goodpasture's syndrome when the aforementioned requirements are met is categorized accordingly: III/2B; I/1C or I/1B.

\section{ASFA Category II}

In renal diseases qualified in the II ASFA category, TA is a second-line therapy, which means it should be applied when the first line of treatment fails.

An example in this case is atypical hemolytic uremic syndrome. In case of the presence of $\mathrm{H}$ factor autoantibodies $\mathrm{I} / 2 \mathrm{C}$ category is recommended, while if diarrhea associated with hemolytic uremic syndrome (HUS) occurs, category IV/1C applies.

New Options of Apheresis in Renal Diseases
The other example in this category is acute symptomatic cryoglobulinemia. The ASFA recommends IA in place of TA; however, the recommendation has a slightly lower grade - II/2B in comparison to TA I/2A.

\section{ASFA Category III}

There is no optimal recommendation for the use of TA in this category. Therefore, the decision to apply any option of apheresis should be individual and depend on clinical state of the patient. Application of TA in Goodpasture syndrome is an example of such approach. It is categorized as ASFA category III grade $2 \mathrm{~B}$. The use of TA depends not only on the degree of renal function, but also on the renal biopsy results. Thus, patients suffering from the Goodpasture syndrome and renal dysfunction (creatinine $\geq 6 \mathrm{mg} / \mathrm{dl}$ ) or dialysis dependence at the time of eligibility for treatment are not positive candidates for the renal function improvement. However, if the preliminary biopsy finds the glomerular damage to be reversible, even while creatinine level in plasma is high ( $\geq 6 \mathrm{mg} / \mathrm{dl}$ ), application of TA may cause the improvement of clinical state.

Another example is immunocomplex RPGN, categorized as category III/2B indication to plasmapheresis. Nevertheless, if the patient shows symptoms of generalized myositis, this method may be ineffective (category IV/2C). It seems prudent to point out that a patient with myositis may equally benefit from leukocytapheresis (category IV/2C).

\section{ASFA Category IV}

This category consists of primary and secondary renal diseases in which TA is ineffective or even harmful. An example of such a disease is systemic amyloidosis for which TPE is recommended, but for ASFA category IV with grade $2 \mathrm{C}$, meaning the reference is based on results of observational research or experts opinion, not the controlled multicenter studies.

Another example is SLE. Lupus nephritis has, according to the ASFA, a category IV and grade $1 \mathrm{~B}$ recommendation, while severe extra renal manifestations of SLE have slightly higher TPE recommendation - category II, grade 2C.

Aforementioned 2013 recommendations convey two significant messages. Firstly, the indications for TA depend on the clinical state of the patient, his renal function and dialysis dependence, like for example, in RPGN, both in ANCA-associated mechanism and in anti-GBM-associated mechanism. The recommendations in this aspect overlap those of 2010 (5th edition of ASFA), which were 
formulated under the guidance of Szczepiorkowski et al. [30]. Secondly, the current recommendations include new options of apheresis also when it comes to renal diseases. According to the ASFA, acute cryoglobulinemia should be treated by TP but alternatively IA could be applied, although under significantly lower category and recommendation (category $\mathrm{II} / 2 \mathrm{~B}$ ). Another example is immunocomplex RPGN with generalized muscle damage, where leukocytapheresis is recommended on the same level as classic TA (category IV/2C). Nephrogenic systemic fibrosis may be treated with erythrocytapheresis, which is recommended equally to classic plasmapheresis (III/2C).

Authors of the latest ASFA recommendations point also to their limitations. Firstly, recommendations are unstable and depend on advancement of the research on pathogenesis and treatment of the diseases they concern, as well as the technological progress of new TPA options.

Secondly, these recommendations do not take into account the urgency level of procedure, that is, emergently, urgently or routinely. The ASFA committee considers the execution of the treatment a decision that is dependent not only on the published recommendations, but also on the clinical state of the patient, technical capabilities of the center responsible for the treatment and the availability of alternative forms of treatment in case of resignation from plasmapheresis or its options.

Shaz et al. [29] at the same time published contemporary criteria for optimal apheresis in form of following canons: what does the patient most likely have (diagnosis); is the diagnosis amenable to treatment with TA; what is the harm-versus-benefit ratio of TA in this patient and external issues associated with retrieving this treatment?

Those criteria constitute a modern version of Bruce McLeod's paper from 2002. McLeod, the longtime president of the ASFA Committee pinpoints the following rules for optimal apheresis: plausible pathogenesis, better blood, perkier patients.

The rules were as follows.

'Plausible pathogenesis implies that we should have a secure understanding of the disease process, and that this understanding should suggest a clear rationale for the TA modality to be applied. Straightforward examples of this are disease states known to be 'caused' by an excess of a circulating antibody. Such knowledge provides a clear rationale for therapeutic cell or antibody removal by apheresis.

Better blood simply means that we should have clear evidence that the abnormality that makes apheresis plau- sible is meaningfully corrected by TA. This concept is closely linked to plausibility because both common sense and the weight of experience indicate that only a certain kind of plasma constitution can be profitably manipulated by TPE.

The third criterion, perkier patients, means that there should be strong evidence that TA confers benefit that is clinically worthwhile and not just statistically significant' [31].

Bruce McLeod warns, meanwhile, that if those criteria were to be treated loosely we could face a situation similar to that of our predecessors who 200 years ago uncritically employed the instructions of Dr. Benjamin Rush, an influential leader of the fledgling American medical community known, among other things, for his enthusiasm for bloodletting as a therapy. He prescribed it indiscriminately. The case of George Washington is an example of the predominant influence Rush had on minds and practices of his followers. Washington, who was most probably suffering from acute bacterial epiglottitis, was bled over $40 \%$ of his blood volume (almost $2.5 \mathrm{l}$ ) within an 8 -hour period on the last day of his life.

Modern medical science would judge this treatment as ineffective and a waste of time and effort. Nevertheless, in medical writings of the 20th and 21st century, bloodletting was still recommended in different clinical cases. For instance, a medical handbook 'Kidney diseases: a companion to medical practitioners and students' penned by Max Rosenberg in the beginning of the 20th century and translated to Polish in 1930 brings us the following recommendation.

'In case of severe symptoms of uremia, bloodletting gives the patient a significant, although passing relief, similarly and from the same reasons as in case of acute uremia. Having in mind more pronounced anemia, bloodletting in acute uremia has to be done carefully and with restraint: in case of severe anemia, not more than $300 \mathrm{~cm} 3$ '. As a point of interest, only 15 years separate this publication by Max Rosenberg and first successful hemodialysis treatment conducted by Willem Kolff in 1945 on a 67-year-old diabetic patient with irreversible renal failure, who was then undergoing dialysis for next 7 years [32].

The question that presents itself here is if and how long will it take new options of apheresis to become modern standards of treatment. Publications of last 15 years brought us merely a handful of articles dealing with this problem - mostly reviews but also observational studies or case reports [25, 33-41].

Reeves and Winters in their 2014 article entitled 'The mechanisms of action in plasma exchange' pointed out 
the fact that despite the 100 years of major technical development since the first experimental apheresis that was conducted by Dr. John Abel [42] in 1914 on uremic dogs, the mechanism behind the treatment and clinical efficacy of PLEX has still not been fully examined. Authors enumerate key issues concerning plasmapheresis, which to this day remain unanswered.

- 'how does the removal of autoantibodies explain the effect of PLEX when antibody titres do not correlate with disease severity/symptoms in many autoimmune disorders?

- how does PLEX produce therapeutic effects in disorders where the presumed pathological autoantibodies are outside of the vascular compartment and therefore not accessible to the treatment, such as in multiple sclerosis and neuromyelitis optica?

- does PLEX alter the immune system?

- if PLEX alters the immune system, such as increasing T regulatory cells, increasing $T$ suppressor response and altering the Th1/Th2 ratio, what is the mechanism behind these changes? Is it the removal of cytokines, removal if antibodies, generation of cytokines through interaction with the apheresis device or are the alterations due to cellular contact with the device?

- is the removal of cytokines, given their short half-lives and large volumes of distribution, factors predicting poor removal, a component of the effect of PLEX?

- are the mechanisms of action different for centrifugation-based and filtration-based PLEX devices given the substantial differences between these techniques?' [43].
Another important problem stems from the high cost of the procedure and still restricted availability of plasmapheresis even in the highly developed countries [44].

Reeves and Winters [43] therefore conclude that there still is a need for intensive controlled clinical studies on plasmapheresis and its new options. Without significant progress in the current practice of apheresis, it will begin to resemble the indiscriminate use of bloodletting to remove unspecified evil humor, which was advocated 200 years ago by Dr Benjamin Rush [31].

\section{Conclusion}

Plasmapheresis and its new options became important tools in the therapy of primary and secondary autoimmune renal diseases. Limited practical use of the method stems mainly from the lack of controlled, multicenter clinical studies as well as the disproportion between a significant technological development and still insufficient knowledge of the method's pathomechanism. It is to be hoped that this new century, thanks to increasingly applied proteomics and molecular biology achievements, will bring on the breakthrough in clinical application of apheresis and its new options, particularly in autoimmune renal diseases.

\section{Disclosure Statement}

Authors have no relevant financial or nonfinancial relationships to disclose.

\section{References}

1 Yokoyama H, Wada T, Furuichi K: Immunomodulation effects and clinical evidence of apheresis in renal diseases. Ther Apher Dial 2003;7:513-519.

2 Kenneth H, Sumak M, Gail A: Therapeutic plasma exchange. N Engl J Med 1984;22:762768.

3 Lockwood CM, Boulton-Jones JM, Lowenthal RM, et al: Recovery from Goodpasture's syndrome after immunosuppressive treatment and plasmapheresis. Br Med J 1975;2:252-254.

4 Kaplan AA: Apheresis for renal disease. Ther Apher 2001;5:134-141.

5 Wańkowicz Z, Tymowicz J, Korsak J: [Therapeutic plasma exchanges with cyclophosphamide in the treatment of rapidly progressing glomerulonephritis]. Pol Merkur Lekarski 1998;5:304-306.

6 Yokoyama H, Wada T, Zhang W, et al: Advances in apheresis therapy for glomerular diseases. Clin Exp Nephrol 2007;11:122127.

7 Agishi T: Birth of the concept and the development of extracorporeal immunomodulation. Ther Apher 2002;6:312-316.

8 McLeod BC: Apheresis: Priciples and Practice, ed 2. AABB Press, 2003.

9 Brecher ME: Plasma exchange: why we do what we do. J Clin Apher 2002;17:207-211.

10 Brecher ME: Technical Manual, ed 1. AABB Press, 2005.

11 McLeod BC, Triluzi DJ: Therapeutic Apheresis: A Physician's Handbook, ed 1. AABB Press, 2005.

12 Galel SA, Nguyen DD, Fontaine MJ, et al: Transfusion medicine. Therapeutic Apheresis; in Greer JP, Foerster J, Rodgers G (eds): Wintrobe's Clinical Hematology. Lippincolt Williams \& Wilkins, 2008, pp 711712.
13 Theunissen K, Bracke T, Berckmans D, et al: Birth of a new generation of therapeutic apheresis platforms: interim analysis of a routine use experience with the spectra optia. J Clin Apher 2007;22:69.

14 Lefevre PA, Poullin P: Therapeutic plasma exchange with the new continous blood flow separator spectra optia. J Clin Apher 2008;23: 43-48.

15 Siami GA, Siami FS: Membrane plasmapheresis in the United States: a review over the last 20 years. Ther Apher 2001;5:315-320.

16 Siami GA, Siami FS: Current topics on cryofiltration technologies. Ther Apher 2001;5: 283-286.

17 Deodhar A, Allen E, Daoud K, et al: Vasculitis secondary to staphylococcal protein A immunoadsorption (Prosorba column) treatment in rheumatoid arthritis. Semin Arthritis Rheum 2002;32:3-9. 
18 Iglesias J, D’Agati VD, Levine JS: Acute glomerulonephritis occurring during immunoadsorption with staphylococcal protein A column (Prosorba). Nephrol Dial Transplant 2004;19:3155-3159.

19 Ronco C, Brendolan A, Lonnemann G, et al: A pilot study of coupled plasma filtration with adsoption in septic shock. Crit Care Med 2002;30:1250-1255.

20 Berlot G, Tomasini A, Agbedjro A: Coupled plasma filtration - adsoption; in Vincent JL (ed): Annual Update in Intensive Care and Emergency Medicine. Springer-Verlag, 2012, pp 653-658.

21 Bosch X, Poch E, Grau JM: Rhabdomyolysis and acute kidney injury. N Engl J Med 2009; 361:62-72.

22 McLeod B, Sniecinski I, Ciavarella D: Frequency of immediate adverse effects associated with therapeutic apheresis. Transfusion 1999;39:282-288.

23 McLeod BC, Price TH, Weinstein R (eds): Apheresis: Principles and Practice. Bethesda, AABB Press, 2003, pp 268-272.

24 Millwad PA, Bandarenko N, Brecher ME: Thrapeutic apheresis basic principles and practical aspects; in Hillyer CD, Silberstein LE, Ness PM (eds): Blood Banking and Transfusion Medicine: Basic Principles and Practice. Churchill Livingstone Elsevier, 2007, pp 727-736.

25 Nakanishi T, Suzuki N, Kuragano T, et al: Current topics in therapeutic plasmapheresis. Clin Exp Nephrol 2014;18:41-49.

26 Szczepiorkowski ZM, Shaz BH, Bandarenko $\mathrm{N}$, et al: The new approach to assignment of ASFA categories - introduction to the fourth special issue: clinical applications of therapeutic apheresis. J Clin Apher 2007;22:96-105.
27 Guyatt GH, Cook DJ, Jaeschke R, et al: Grades of recommendation for antithrombotic agents: American college of chest physicians evidence-based clinical practice guidelines (8th edition). Chest 2008;133(6 suppl):123S$131 \mathrm{~S}$.

28 Schwartz J, Winters JL, Padmanabhan A, et al: Guidelines on the use of therapeutic apheresis in clinical practice-evidence-based approach from the writing committee of the American Society for Apheresis: the sixth special issue. J Clin Apher 2013;28:145-284.

29 Shaz BH, Schwartz J, Winters JL: How we developed and use the American Society for Apheresis guidelines for therapeutic apheresis procedures. Transfusion 2014;54:17-25.

30 Szczepiorkowski ZM, Winters JL, Bandarenko N, et al: Guidelines on the use of therapeutic apheresis in clinical practice-evidencebased approach from the American Society for Apheresis. J Clin Apher 2010;25:83-177.

31 McLeod BC: An approach to evidence-based therapeutic apheresis. J Clin Apher 2002;17: 124-132.

32 Wańkowicz Z: The role of technological progress vs. accidental discoveries and clinical experience. Med Sci Monit 2013;19:984-992.

33 Stenvinkel P, Alvestrand A, Angelin B, et al: LDL-apheresis in patients with nephrotic syndrome: effects on serum albumin and urinary albumin excertion. Eur J Clin Invest 2000;30:866-870

34 Haas M, Böhmig GA, Leko-Mohr Z, et al: Peri-operative immunoadsorption in sensitized renal transplant recipients. Nephrol Dial Transplant 2002;17:1503-1508.

35 Sułowicz W, Stompór T: LDL-apheresis and immunoadsorption: novel methods in the treatment of renal diseases refractory to conventional therapy. Nephrol Dial Transplant 2003;18(suppl 5):v59-v62.
36 Yamagata K, Hirayama $\mathrm{K}$, Mase $\mathrm{K}$, et al: Apheresis for MPO-ANCA-associated RPGN-indications and efficacy: lessons learned from Japan nationwide survey of RPGN. J Clin Apher 2005;20:244-251.

37 Yokoyama H, Wada T, Zhang W, et al: Advances in apheresis therapy for glomerular diseases. Clin Exp Nephrol 2007;11:122-127.

38 Sanchez AP, Ward DM: Therapeutic apheresis for renal disorders. Semin Dial 2012;25: 119-131.

39 Miyata $\mathrm{H}$, Uno K, Ono T, et al: Low density lipoprotein apheresis ameliorates interferon- $\gamma$ production in patients with nephrotic syndrome. Ther Apher Dial 2012;16:189-194.

40 Hickstein H, Koball S, Lehmann R, et al: ABO incompatible kidney transplantation using unspecific immunoadsorption. Transfus Apher Sci 2014;50:263-266.

41 Kobayashi S, Tamura N, Tsuda H, et al: Immunoadsorbent plasmapheresis for a patient with antiphospholipid syndrome during pregnancy. Ann Rheum Dis 1992;51:399401.

42 Abel JJ, Rowntree LG, Turner BB: Plasma removal with return of corpuscles (plasmaphaeresis): first paper. J Pharmacol Exp Ther 1914; 5:625-641.

43 Reeves HM, Winters JL: The mechanisms of action of plasma exchange. Br J Haematol 2014;164:342-351.

44 Cybulsky AV, Walsh M, Knoll G, et al: Canadian society of nephrology commentary on the 2012 KDIGO clinical practice guideline for glomerulonephritis: management of glomerulonephritis in adults. Am J Kidney Dis 2014;63:363-377. 